\title{
GO Shaping of Omnidirectional Dual-Reflector Antennas with Arbitrary Main-Beam Direction in Elevation Plane by Connecting Conic Sections
}

\author{
Rafael A. Penchel, ${ }^{1}$ Sandro R. Zang, ${ }^{2}$ José R. Bergmann $\left(\mathbb{D},{ }^{3}\right.$ and Fernando J. S. Moreira ${ }^{4}$ \\ ${ }^{1}$ São Paulo State University (UNESP)-São João da Boa Vista, 13876-750 São João da Boa Vista, SP, Brazil \\ ${ }^{2}$ Department of Mechatronics and Telecommunication Engineering, UFSJ, Campus Alto Paraopeba (C.A.P), 36.420-000 Ouro \\ Branco, MG, Brazil \\ ${ }^{3}$ CETUC (Center for Telecommunications Studies), Pontifical Catholic University of Rio de Janeiro, 22453-900 Rio de Janeiro, \\ RJ, Brazil \\ ${ }^{4}$ Department of Electronics Engineering, Federal University of Minas Gerais, 31270-901 Belo Horizonte, MG, Brazil
}

Correspondence should be addressed to José R. Bergmann; bergmann@cetuc.puc-rio.br

Received 28 June 2017; Revised 10 November 2017; Accepted 11 December 2017; Published 4 March 2018

Academic Editor: Paolo Burghignoli

Copyright (C) 2018 Rafael A. Penchel et al. This is an open access article distributed under the Creative Commons Attribution License, which permits unrestricted use, distribution, and reproduction in any medium, provided the original work is properly cited.

\begin{abstract}
This work discusses an alternative geometrical optics (GO) technique to synthesize omnidirectional dual-reflector antennas with uniform aperture phase distribution together with an arbitrary main-beam direction for the antenna radiation pattern. Sub- and main reflectors are bodies of revolution generated by shaped curves defined by local conic sections consecutively concatenated. The shaping formulation is derived for configurations like ADC (axis-displaced Cassegrain) and ADE (axis-displaced ellipse) omnidirectional antennas. As case studies, two configurations fed by a TEM coaxial horn are designed and analyzed by a hybrid technique based on mode matching and method of moments in order to validate the GO shaping procedure.
\end{abstract}

\section{Introduction}

The spectral congestion in urban centers and large data rates required by the next generation technology $(5 \mathrm{G})$ of broadband mobile communication have forced the development of systems operating at higher frequencies [1]. For radio channels between 28 and $38 \mathrm{GHz}$, highly efficient-shaped omnidirectional reflector antennas can be suited to provide broadband communications with a compact design.

For high-gain or omnidirectional antennas, several works have dealt with the shaping of circularly symmetric dual-reflector for prescribed equiphase field distribution at the aperture by solving an ordinary differential equation derived from geometrical optics (GO) principles [2-4]. For collimated beam, [5] presents an alternative GO shaping technique based on the representation of the reflector generatrices by concatenated local conic sections. There, the authors employ rectangular coordinates to describe the local conic sections representing the reflectors' generatrices, leading to a set of nonlinear algebraic equations. It represented an improvement over traditional methods, although biparabolic sufaces have already been employed to locally represent the reflector in the GO synthesis of offset dual-reflector antennas [6]. Further improvements were presented in [7], where, alternatively, the authors use polar coordinates to represent the conic sections, leading to a one-step iterative procedure with simple linear algebraic equations. In [8], this alternative technique was applied to shape the main reflector of omnidirectional dual-reflector antennas to control power distribution along the vertical plane, while in [9], it was applied to shape both reflectors for maximum directivity along the horizon. When compared to the techniques based on the solution of differential equations, the combination of confocal conic sections proved to be simpler and more efficient for many circularly symmetric dual-reflector arrangements. 
The present work generalizes the formulation presented in [9] to attain an arbitrary main-beam direction in the elevation plane. The GO synthesis technique is described in Section 2. To illustrate the procedure, Section 3 presents design examples of ADE (axis-displaced ellipse) and ADC (axis-displaced Cassegrain) configurations, shaped for uniform phase and amplitude distribution at conical apertures. Besides the uniform power distribution for maximum gain, a tapered amplitude distribution is explored to control the side lobe levels behind the main reflector. A rigorous analysis combining the mode matching technique (MMT) and the method of moments (MoM) [10] is employed to validate the synthesis.

\section{GO Shaping Technique}

The shaped dual-reflector configurations are composed of two circularly symmetric (bodies of revolution) reflectors with a common symmetry axis (the $z$-axis). The subreflector is generated by a combination of local conic sections $S_{n}(n=1,2,3, \ldots, N)$, sequentially concatenated to each other. Each $S_{n}$ has a (common) focus at the origin $(O)$ and the other $P_{n}$ at a caustic region, as illustrated in Figures 1 and 2 for ADE- and ADC-like configurations, respectively. The focus $P_{n}$, after rotation around the symmetry axis, defines a ring caustic. For the ADE configuration, $S_{n}$ is an ellipse and its ring caustic is real (Figure 1), while for the ADC configuration, $S_{n}$ is a hyperbola and the ring caustic is virtual (Figure 2). The main reflector is generated by parabolas $M_{n}(n=1,2,3, \ldots, N)$ with a focus at $P_{n}$ and a common axial tilt angle $\gamma$ in the elevation plane. The feed horn, with its phase center at $O$, has a circularly symmetric radiation pattern $G_{F}\left(\theta_{F}\right)$, where $\theta_{F}$ is the angle between the feed ray and the $z$-axis. From a GO perspective, the rays emanating from $O$ are reflected by $S_{n}$ toward $M_{n}$. After the second reflection, the rays arrive at a conical aperture, parallel to each other (in the elevation plane), which provides an aperture illumination with a uniform phase distribution and a prescribed circularly symmetric power density $G_{A}\left(x^{\prime}\right)$, where $x^{\prime}$ and $z^{\prime}=z_{A}^{\prime}$ are the aperture coordinates in the auxiliary system $x^{\prime}, z^{\prime}$ (see Figures 1 and 2).

Each pair of conics $S_{n}$ and $M_{n}$ are determined by an iterative process where the feed ray direction $\theta_{F}$ is uniformly varied from $\theta_{F 0}=0(n=0)$ up to the subreflector edge angle $\theta_{F N}=\theta_{E}(n=N)$. The initial condition $(n=0)$ is imposed by the locations of the subreflector vertex $Q$ (at the symmetry axis, at a known distance $r_{F 0}=V_{S}$ from $O$ ) and the corresponding main reflector point $U$ (for the $\mathrm{ADE}$ ) or $L$ (for the ADC), which must be specified a priori. At each $n$th step, the subreflector section $S_{n}$, which is limited by the tube of rays within $\theta_{F n-1} \leq \theta_{F} \leq \theta_{F n}$, is uniquely determined by its interfocal distance $2 c_{n}$ (i.e., the distance between $O$ and $P_{n}$ ), eccentricity $e_{n}$, and axial tilt angle $\beta_{n}$ with respect to the $z$-axis. The corresponding main reflector section $M_{n}$ is solely determined by its focal distance $F_{n}$, as its axis tilt angle $\gamma$ is known a priori. Essentially, $\gamma$ defines the antenna mainbeam direction in the elevation plane, being an input parameter. Consequently, four equations are required to determine $c_{n}, e_{n}, \beta_{n}$, and $F_{n}$.

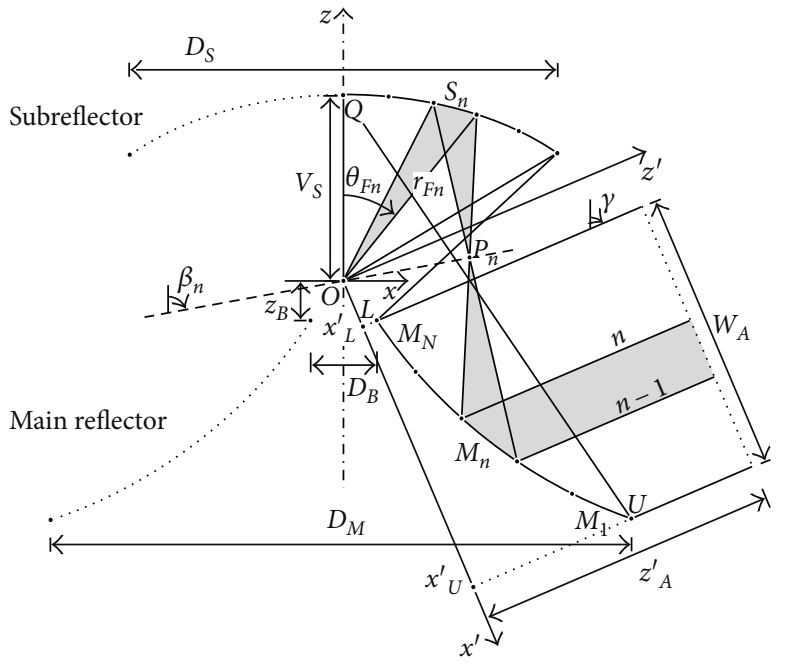

FIgURE 1: Geometry of the shaped omnidirectional ADE-like antenna.

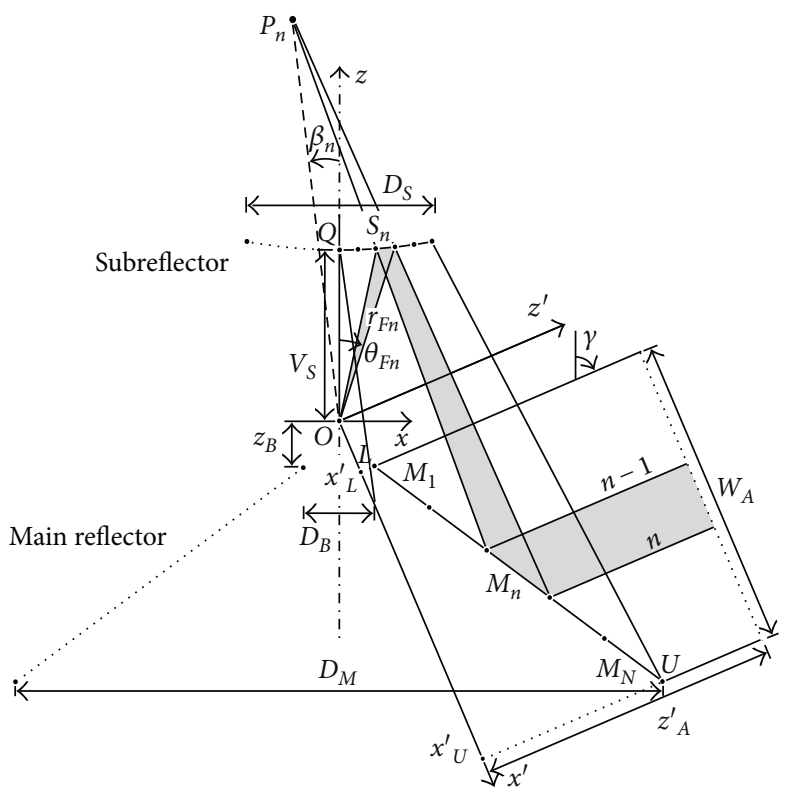

FIGURE 2: Geometry of the shaped omnidirectional ADC-like antenna.

The iterative process closely follows that of [7]. The first equation comes from the polar representation of $S_{n}$, which gives $r_{F}$ (the distance from $O$ to $S_{n}$ ) as function of $\theta_{F}$ :

$$
r_{F}=\frac{a_{n}}{b_{n} \cos \theta_{F}+\underbrace{d_{n} \sin \theta_{F}-1}}, \quad \theta_{F n-1} \leq \theta_{F} \leq \theta_{F n},
$$

where

$$
\begin{aligned}
& a_{n}=c_{n}\left(e_{n}-\frac{1}{e_{n}}\right), \\
& b_{n}=e_{n} \cos \beta_{n}, \\
& d_{n}=e_{n} \sin \beta_{n} .
\end{aligned}
$$


Equation (1) is general and may represent an ellipse $\left(0<e_{n}<1\right)$ or a hyperbola $\left(\left|e_{n}\right|>1\right)$. Notice that sections $S_{n-1}$ and $S_{n}$ share a common point at their junction. So, with $r_{F n-1}$ known from the previous step $n-1$, the first equation of the $n$th step is

$$
r_{F n-1}=\frac{a_{n}}{b_{n} \cos \theta_{F n-1}+d_{n} \sin \theta_{F n-1}-1} .
$$

To obtain a uniform phase distribution over the conical aperture, the optical path from $O$ to the aperture (at $z^{\prime}=z_{A}^{\prime}$, as illustrated in Figures 1 and 2) must be constant (equal to $\ell_{o}$ and known a priori). With the help of the conic equations of $S_{n}$ and $M_{n}$, one obtains the second shaping equation:

$$
\ell_{o}=\frac{2 c_{n}}{e_{n}}+2 F_{n}-2 c_{n} \cos \left(\beta_{n}-\gamma\right)+z_{A}^{\prime} .
$$

The constant optical path $\ell_{o}$ also provides a simpler mapping relation between the feed ray $\theta_{F}$ and the corresponding aperture coordinate $x^{\prime}$ :

$$
\frac{x^{\prime}}{\ell_{o}}=\frac{\left(2 a_{n} / \ell_{o}\right)+1+b_{n}^{\prime}-d_{n}^{\prime} \cot \left(\left(\theta_{F}-\gamma\right) / 2\right)}{d_{n}^{\prime}+\left(b_{n}^{\prime}-1\right) \cot \left(\left(\theta_{F}-\gamma\right) / 2\right)}, \quad \theta_{F n-1} \leq \theta_{F} \leq \theta_{F n},
$$

where

$$
\begin{aligned}
& b_{n}^{\prime}=e_{n} \cos \left(\beta_{n}-\gamma\right), \\
& d_{n}^{\prime}=e_{n} \sin \left(\beta_{n}-\gamma\right) .
\end{aligned}
$$

So, observing once more that consecutive conic sections share a common point, the third shaping equation is obtained from (5) at $\theta_{F}=\theta_{F n-1}$ :

$$
\frac{x_{n-1}^{\prime}}{\ell_{o}}=\frac{\left(2 a_{n} / \ell_{o}\right)+1+b_{n}^{\prime}-d_{n}^{\prime} \cot \left(\left(\theta_{F n-1}-\gamma\right) / 2\right)}{d_{n}^{\prime}+\left(b_{n}^{\prime}-1\right) \cot \left(\left(\theta_{F n-1}-\gamma\right) / 2\right)},
$$

where $x_{n-1}^{\prime}$ is known from the previous step $n-1$.

The fourth equation also comes from (5), but at $\theta_{F}=\theta_{F n}$. But first, the corresponding aperture coordinate $x_{n}^{\prime}$ must be defined using the conservation of energy inside the bundle of rays departing from $O$ with $\theta_{F n-1} \leq \theta_{F} \leq \theta_{F n}$ and arriving at the conical aperture with $x_{n-1}^{\prime} \leq x^{\prime} \leq x_{n}^{\prime}$ (see Figures 1 and 2):

$$
\int_{\theta_{F n-1}}^{\theta_{F n}} G_{F}\left(\theta_{F}\right) \sin \theta_{F} d \theta_{F}=N_{F} \int_{x_{n-1}^{\prime}}^{x_{n}^{\prime}} G_{A}\left(x^{\prime}\right) d x^{\prime},
$$

where $N_{F}$ is a normalization factor. Once $x_{n}^{\prime}$ is calculated from (8), the fourth shaping equation is then given by

$$
\frac{x_{n}^{\prime}}{\ell_{o}}=\frac{\left(2 a_{n} / \ell_{o}\right)+1+b_{n}^{\prime}-d_{n}^{\prime} \cot \left(\left(\theta_{F n}-\gamma\right) / 2\right)}{d_{n}^{\prime}+\left(b_{n}^{\prime}-1\right) \cot \left(\left(\theta_{F n}-\gamma\right) / 2\right)} .
$$

By substituting (3) and (4) into (7) and (9), one obtains

$$
\begin{aligned}
& f_{1} b_{n}^{\prime}+g_{1} d_{n}^{\prime}=h_{1}, \\
& f_{2} b_{n}^{\prime}+g_{2} d_{n}^{\prime}=h_{2},
\end{aligned}
$$

where

$f_{1}=x_{n-1}^{\prime} \cot \frac{\left(\theta_{F n-1}-\gamma\right)}{2}-\ell_{o}-2 r_{F n-1} \cos \left(\theta_{F n-1}-\gamma\right)$,

$g_{1}=x_{n-1}^{\prime}+\ell_{o} \cot \frac{\left(\theta_{F n-1}-\gamma\right)}{2}-2 r_{F n-1} \sin \left(\theta_{F n-1}-\gamma\right)$,

$h_{1}=x_{n-1}^{\prime} \cot \frac{\left(\theta_{F n-1}-\gamma\right)}{2}+\ell_{o}-2 r_{F n-1}$,

$f_{2}=x_{n}^{\prime} \cot \frac{\left(\theta_{F n}-\gamma\right)}{2}-\ell_{o}-2 r_{F n-1} \cos \left(\theta_{F n-1}-\gamma\right)$,

$g_{2}=x_{n}^{\prime}+\ell_{o} \cot \frac{\left(\theta_{F n}-\gamma\right)}{2}-2 r_{F n-1} \sin \left(\theta_{F n-1}-\gamma\right)$,

$h_{2}=x_{n}^{\prime} \cot \frac{\left(\theta_{F n}-\gamma\right)}{2}+\ell_{o}-2 r_{F n-1}$.

After $b_{n}^{\prime}$ and $d_{n}^{\prime}$ are obtained from (10), $a_{n}$ is directly calculated from (3):

$$
a_{n}=r_{F n-1}\left(b_{n} \cos \theta_{F n-1}+d_{n} \sin \theta_{F n-1}-1\right),
$$

where from (2) and (6),

$$
\begin{aligned}
& b_{n}=b_{n}^{\prime} \cos \gamma-d_{n}^{\prime} \sin \gamma, \\
& d_{n}=b_{n}^{\prime} \sin \gamma+d_{n}^{\prime} \cos \gamma .
\end{aligned}
$$

Then, $c_{n}, e_{n}$, and $\beta_{n}$ are immediately determined from (2). Finally, $F_{n}$ is then obtained from (4). The shaping technique presented here assumes omnidirectional $\mathrm{ADE}$ or ADC configurations, as illustrated in Figures 1 and 2, respectively. However, the technique can be extended to the other omnidirectional configurations [11].

\section{Design Analysis}

To illustrate the GO shaping technique described in Section 2, the first case study is an ADE-like configuration designed with uniform amplitude distribution $\left(G_{A}=\right.$ cte. $)$ over the conical aperture, in order to increase the directivity of the omnidirectional antenna. The feed is a TEM coaxial horn with its radiation pattern described in [8] and modelled as follows in the present synthesis procedure:

$$
\begin{array}{r}
G_{F}\left(\theta_{F}\right)=G_{F 0}\left[\frac{J_{0}\left(k \mu_{i} \sin \theta_{F}\right)-J_{0}\left(k \mu_{e} \sin \theta_{F}\right)}{\sin \theta_{F}}\right]^{2}, \\
0 \leq \theta_{F} \leq \frac{\pi}{2},
\end{array}
$$

where $G_{F 0}$ is a normalization factor, $J_{0}(\cdot)$ is the Bessel function of order 0 , and $\mu_{e}$ and $\mu_{i}$ were adjusted to match the radiation pattern provided by the electromagnetic analysis $\left(\mu_{e}=1.1 \lambda\right.$ and $\left.\mu_{i}=0.3 \lambda\right)$. Figure 3 shows a comparison of the feed radiation pattern given by the MoM analysis and by the model of (14). 


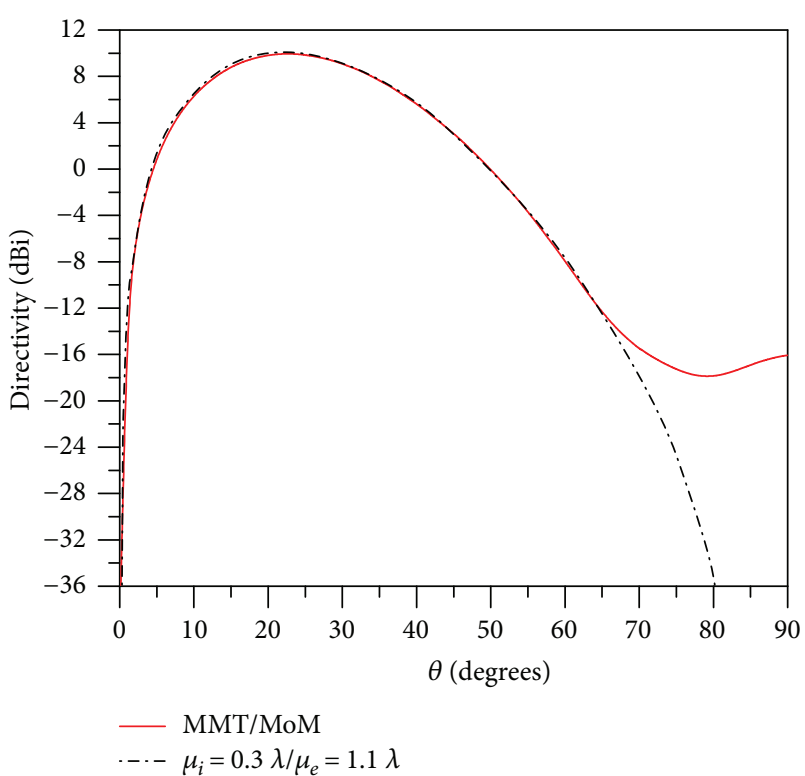

FIgUre 3: Model for the TEM feed radiation pattern.

The initial shaping parameters are defined with the help of a classical ADE configuration, established from the design procedure of [11] with $W_{A}=10 \lambda, D_{M}=24 \lambda, V_{S}=9.5 \lambda$, $D_{B}=2.4 \lambda, z_{B}=0$, and $\gamma=102^{\circ}$. The beam direction $\gamma=$ $102^{\circ}$ was chosen to explore the potentialities of the configurations as base station antennas in point-to-multipoint microwave and millimeter wave radio links. From these input parameters, the elliptical classical subreflector generatrix is obtained with eccentricity $e=0.254$, interfocal distance $2 c=4.546 \lambda$, and axial tilt angle $\beta=61.842^{\circ}$ [11]. Consequently, the projected diameter of the classical subreflector is $D_{S}=18.636348 \lambda$ with an edge angle $\theta_{E}=56.352^{\circ}$, as depicted in Figure 4. The main reflector parabolic generatrix has a focal distance $F=2.917075 \lambda$ and axial tilt angle $\gamma$ $=102^{\circ}$. This classical configuration was used to establish the initial shaping parameters: subreflector vertex $Q$ at $x=0$ and $z=V_{S}=9.5 \lambda$, edge angle $\theta_{E}=56.352^{\circ}$, main reflector point $U$ at $x=D_{M} / 2=12 \lambda$ and $z=-12.519 \lambda$, and aperture width $W_{A}=10 \lambda$. The iterative procedure started $(n=0)$ with the principal optical path $O Q U$ with $\theta_{F}=0$. Consequently, $r_{F 0}=V_{S}=9.5 \lambda$, and from the initial geometry, the constant path length from $O$ to the conical aperture is $\ell_{o}=$ $20.236 \lambda$ for $z_{A}^{\prime}=0$.

Figures 4 and 5 show the classical and shaped reflectors' generatrices, respectively, where one can observe the apparent caustic region between the sub- and main reflectors of the shaped configuration (Figure 5). When the ray tracing in Figures 4 and 5 are compared to each other, it is possible to observe the larger density of rays around the main reflector edges of the shaped antenna (Figure 5) to compensate for the lower horn illumination (see Figure 3 ) toward the subreflector vertex $\left(\theta_{F}=0\right)$ and edge $\left(\theta_{F}=\theta_{E}\right)$.

A full-wave analysis based on the MoM/MMT technique [10] was conducted to validate the shaping procedure. To access the performance of the technique, Figure 6 compares the radiation patterns of the classical ADE configuration

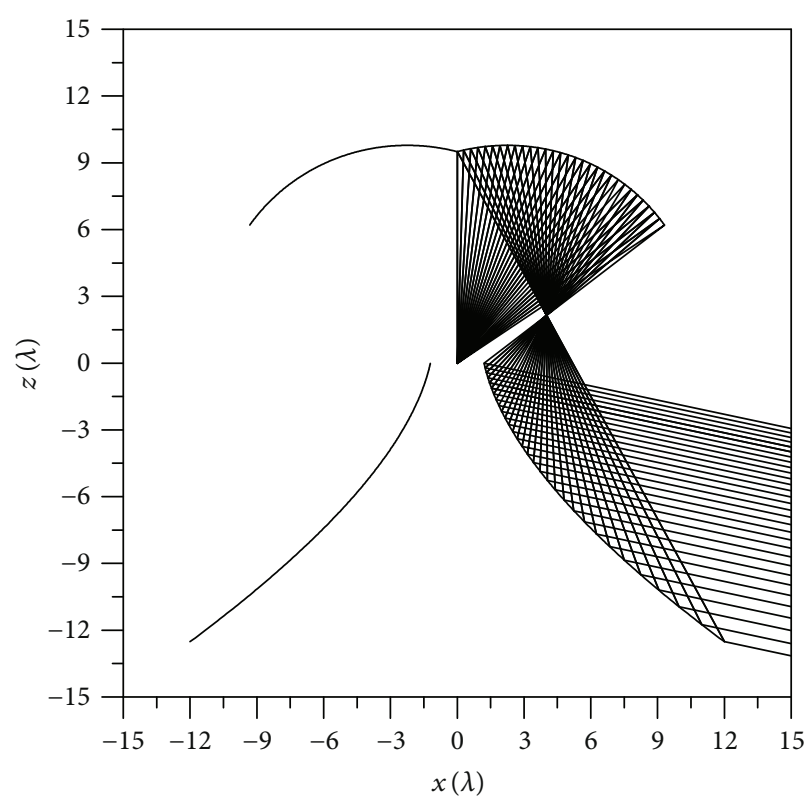

FIgURE 4: Generatrices and ray tracing of the classical omnidirectional ADE antenna.

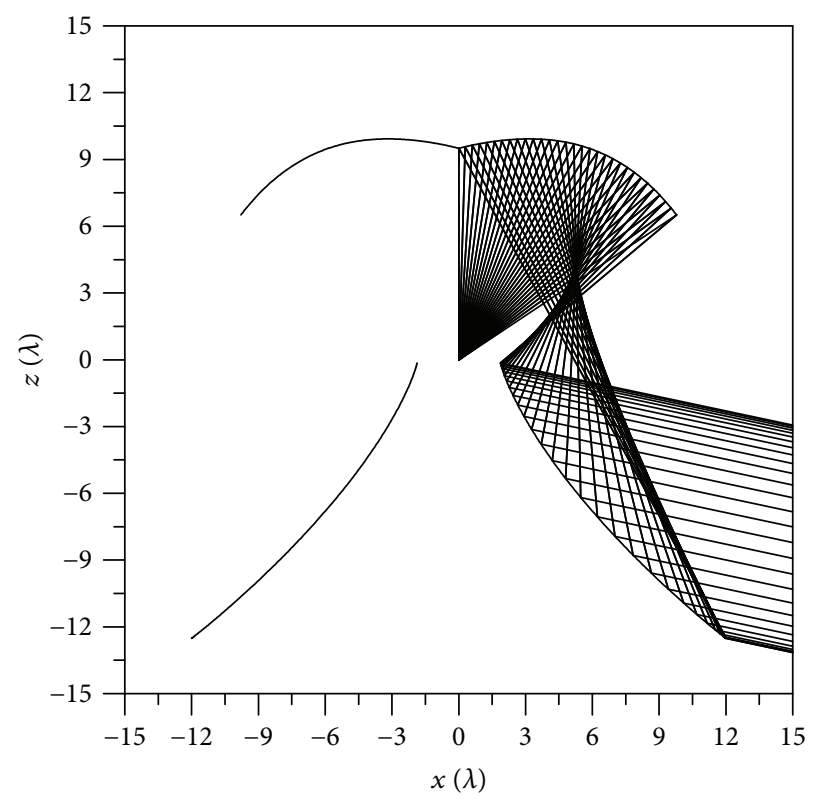

FIgURE 5: Generatrices and ray tracing of the shaped omnidirectional ADE antenna.

provided by the MoM/MMT scheme and by a full-wave commercial simulator (CST Microwave Studio). Figure 7 presents the far-field radiation patterns of the classical (Figure 4) and shaped (Figure 5) configurations. As expected, both antennas have their main-beam peaks at $\theta \approx \gamma=102^{\circ}$. The shaped configuration $\left(\mathrm{D}_{0}=12.79 \mathrm{dBi}, 95 \%\right.$ efficiency) is more directive than the classical one $\left(\mathrm{D}_{0}=11.67 \mathrm{dBi}, 73 \%\right.$ efficiency), as it was designed to have a uniform field distribution over its conical aperture (from a GO perspective). When compared to the classical omnidirectional ADE configuration, the aperture uniform illumination rises the side lobes 


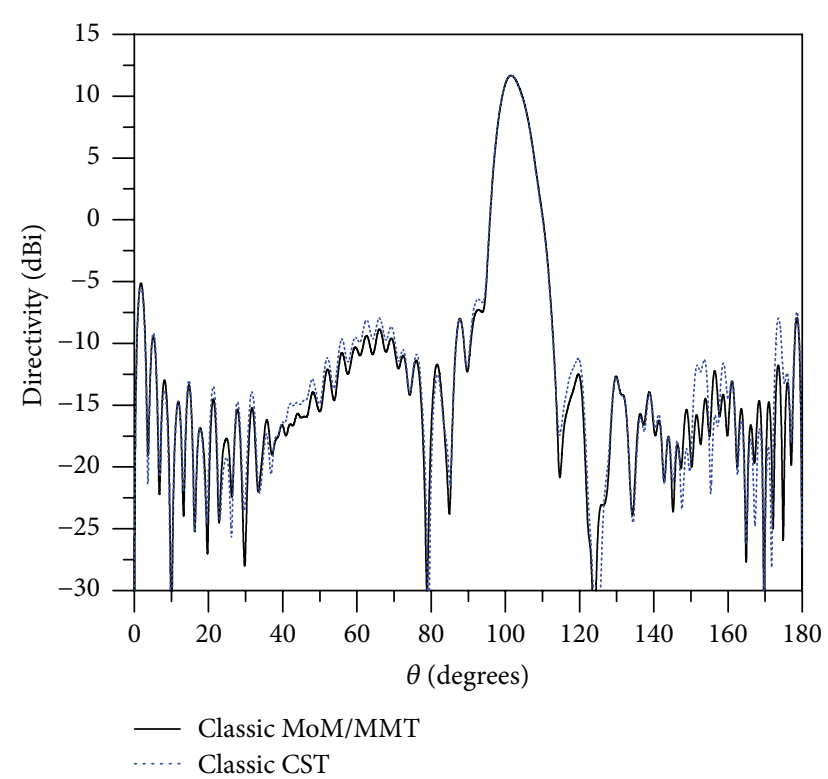

FIGURE 6: MoM/MMT and CST radiation patterns of the classical ADE antennas.

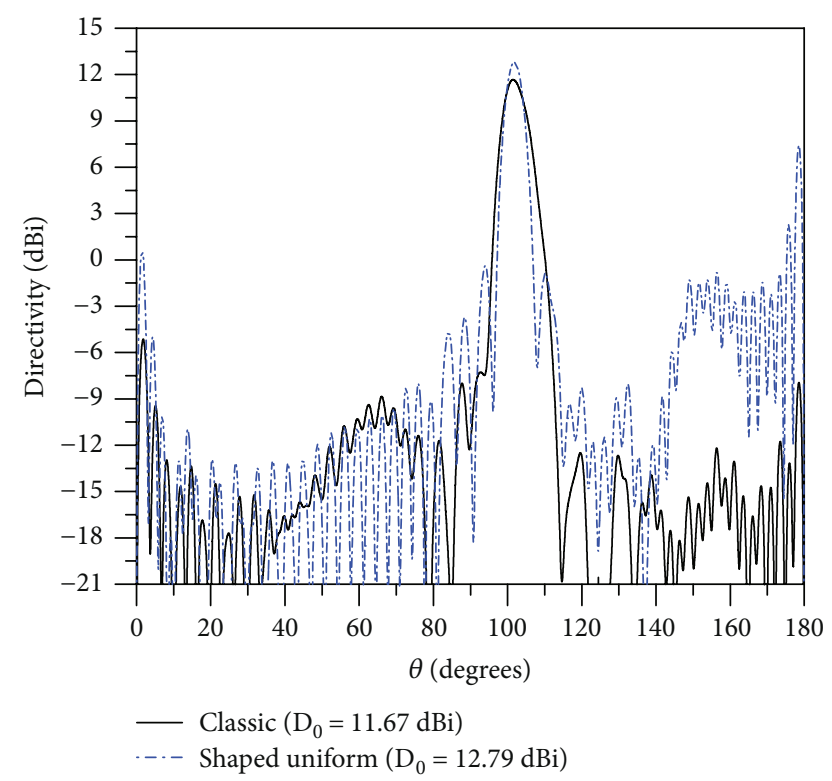

Figure 7: MoM/MMT radiation patterns of the classical and shaped ADE antennas.

close to the main beam, and, due to the increase of the density of rays toward the main reflector edge (see Figure 5), it increases the main reflector spillover and rises the side lobe level behind the main reflector, particularly at $140^{\circ}<\theta<180^{\circ}$ (see Figure 7).

In the second case study, an ADC-like configuration is shaped to provide a uniform aperture illumination, as in the previous case. The initial parameters are established for a classical ADC configuration [11] with dimensions similar to those of the previous classical omnidirectional ADE antenna: $W_{A}=10 \lambda, D_{M}=24 \lambda, V_{S}=10.7 \lambda, D_{B}=2.4$ $\lambda, z_{B}=0$, and $\gamma=102^{\circ}$. These input parameters lead to

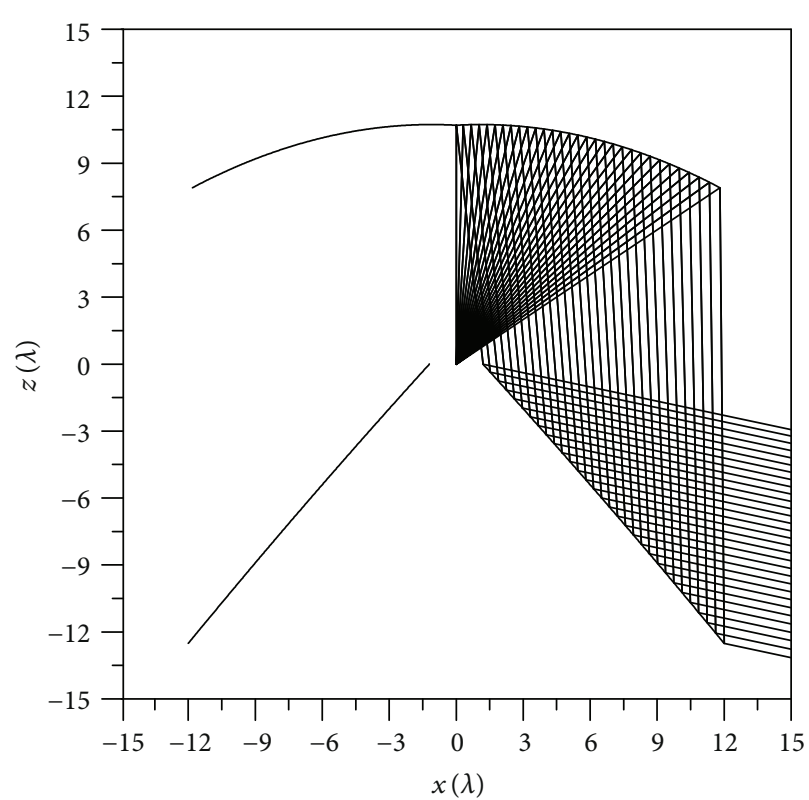

FIgURE 8: Generatrices and ray tracing of the classical omnidirectional ADC antenna.

an elliptical subreflector generatrix with eccentricity $e=$ 0.830880 , interfocal distance $2 c=104.776381 \lambda$, and axial tilt angle $\beta=172.949^{\circ}$. Consequently, the projected subreflector diameter is $D_{S}=23.615148 \lambda$ with edge angle $\theta_{E}=56.199^{\circ}$, as illustrated in Figure 8. The parabolic generatrix of the classical main reflector has a focal distance $F=-35,8047 \lambda$ and an axial tilt angle $\gamma=102^{\circ}$. As mentioned in [11], the negative focal distance $\mathrm{F}$ is associated to a main reflector parabola with reversed curvature and a subreflector with elliptical generatrix (see Figure 8). The classical configuration was employed to establish the initial shaping parameters: subreflector vertex $Q$ at $x=0$ and $z=V_{S}=10.7 \lambda$, edge angle $\theta_{E}=56.199^{\circ}$, main reflector point $L$ at $x=D_{B} / 2=1.2 \lambda$ and $z=0$, and aperture width $W_{A}=10 \lambda$. The iterative procedure started $(n=0)$ with the principal optical path $O Q L$ with $\theta_{F}=0$. Consequently, $r_{F 0}=V_{S}=10.7 \lambda$ and the constant path length from $O$ to the conical aperture is $\ell_{o}=20.2933 \lambda$, for $z_{A}^{\prime}=0$.

Figures 8 and 9 show the sub- and main reflector generatrices of the classical and shaped configurations, respectively, where one may observe the increase in the density of rays around the main reflector edges to compensate for the lower feed illumination at $\theta_{F}=0$ and $\theta_{F}=\theta_{E}$. Again, to access the performance of the MoM/MMT technique, Figure 10 compares the radiation patterns of the classical ADC configuration provided by the MoM/MMT scheme and by a full-wave commercial simulator (CST Microwave Studio). Figure 11 presents the far-field radiation patterns of the classical and shaped configurations obtained from the MoM/MMT technique [10]. Once more, both antennas have their main-beam peaks at $\theta \approx \gamma=102^{\circ}$ and the shaped configuration ( $\mathrm{D}_{0}=12.58 \mathrm{dBi}, 90 \%$ efficiency) is more directive than classical one ( $\mathrm{D}_{0}=11.94 \mathrm{dBi}, 78 \%$ efficiency), as the former was shaped to provide a uniform aperture GO illumination. Once more, one observes the increase of the 


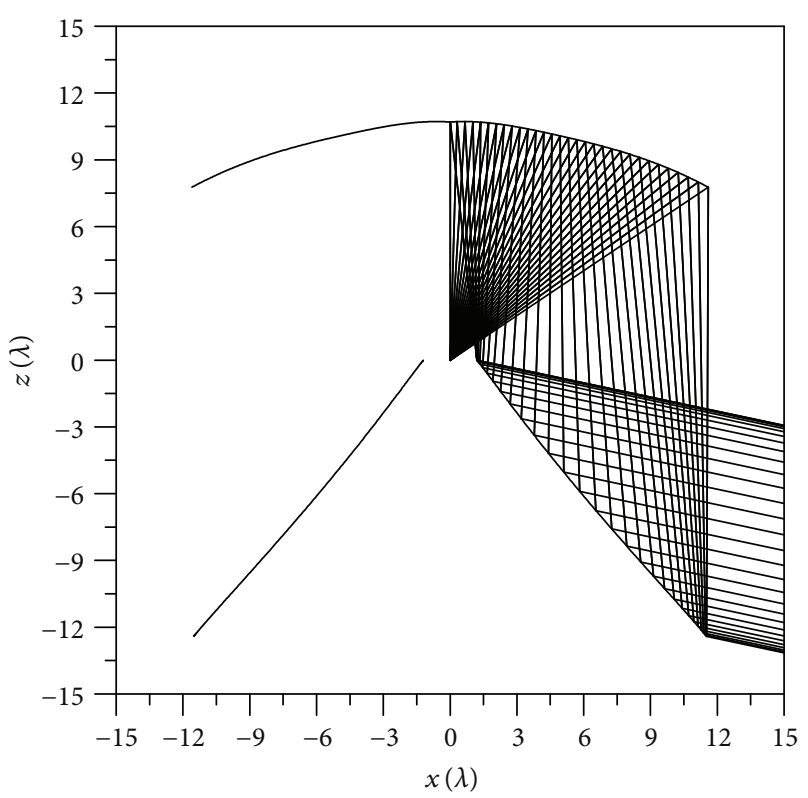

Figure 9: Generatrices and ray tracing of the shaped omnidirectional ADC antenna.

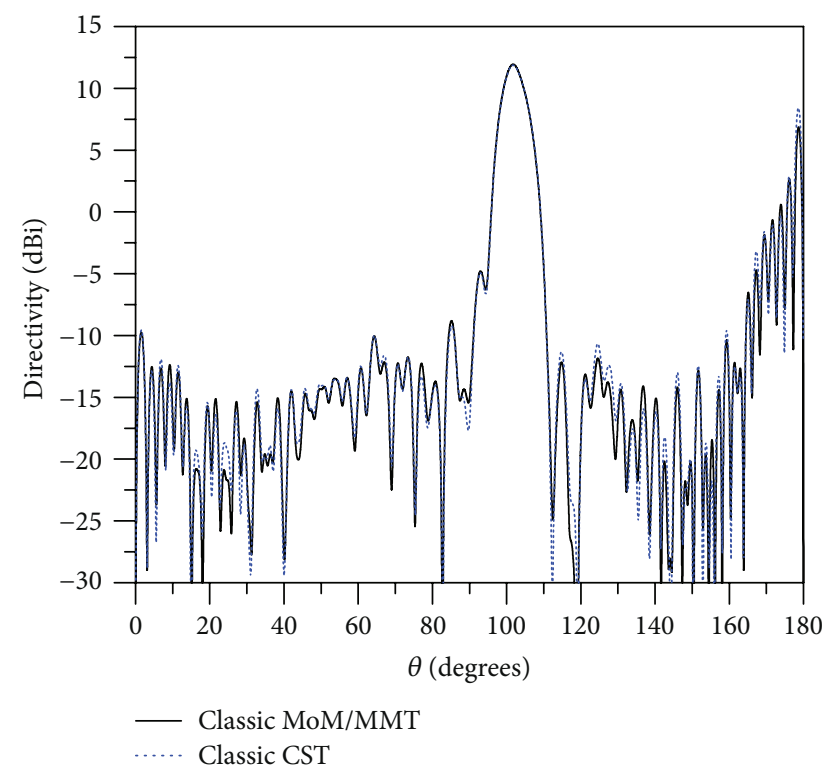

Figure 10: MoM/MMT and CST radiation patterns of the classical and shaped ADE antenna.

side lobe level of the shaped antenna due to the increase of the main reflector spillover.

To investigate the numerical convergence of the proposed technique, surface RMS errors as a function of the number of steps $(N)$ used in the iterative shaping process were simulated. For both sub- and main reflectors, the errors were calculated from the distances between $O$ and the reflector points shared by adjacent generating conics $\left(S_{n-1}\right.$ and $S_{n}$ for subreflectors, $M_{n-1}$ and $M_{n}$ for main reflectors), with the reference being a surface shaped with $N=10^{5}$, a number

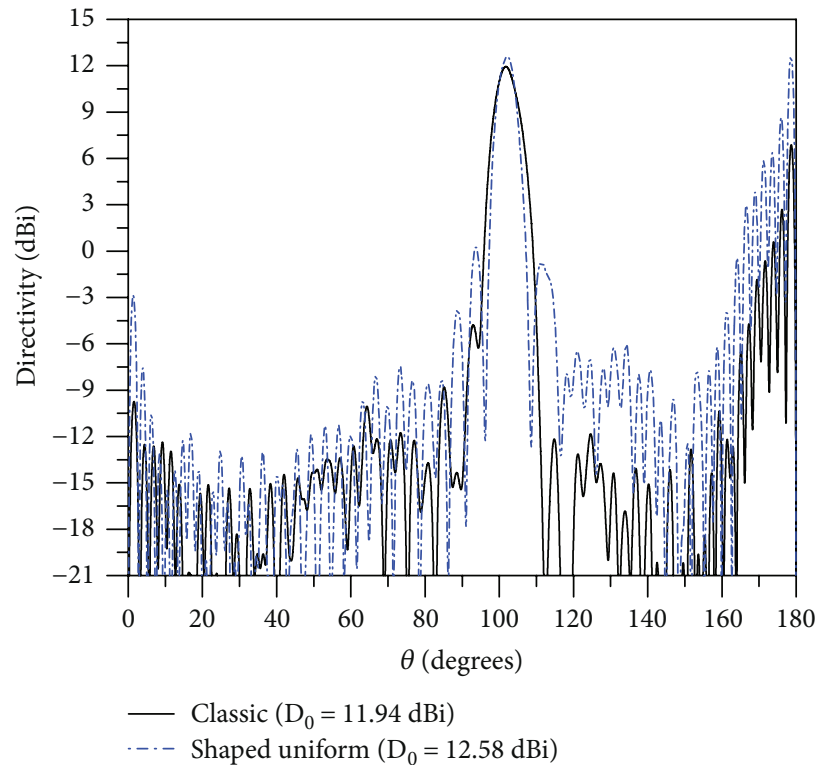

Figure 11: MoM/MMT radiation patterns of the classical and shaped ADC antennas.

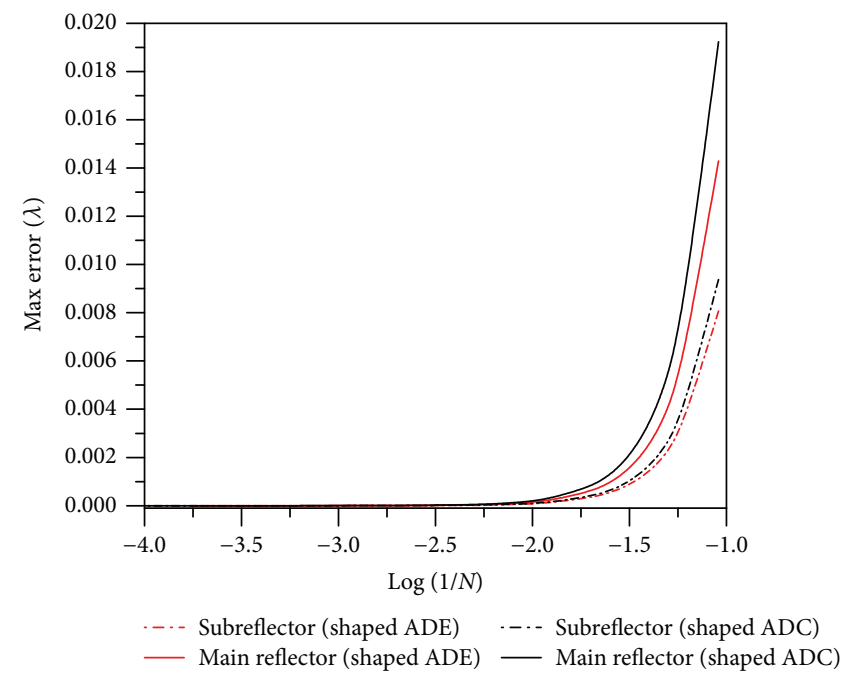

FIgURe 12: Maximum error of shaped ADE and ADC antennas as function of $N$.

large enough to ensure the proper convergence of the reference solution [7]. Figure 12 shows the maximum errors for the shaped sub- and main reflectors of Figures 5 (ADE) and 9 (ADC) as a function of $N$. Due to the cumulative effect, the maximum errors occur for reflector points associated to $\theta_{F}=\theta_{E}$, that is, at the edge of the subreflector and at one of the main reflector extremes. Designs with $N=20$ show maximum error of $0.004 \lambda$ and RMS error of $0.002 \lambda$ for the ADE-like configuration and maximum error of $0.005 \lambda$ and RMS error of $0.003 \lambda$ for the ADC-like configuration.

To illustrate the usefulness and versatility of the shaping technique, the $\mathrm{ADE}$ and $\mathrm{ADC}$ geometries were shaped once more, now to reduce the side lobe levels observed in 


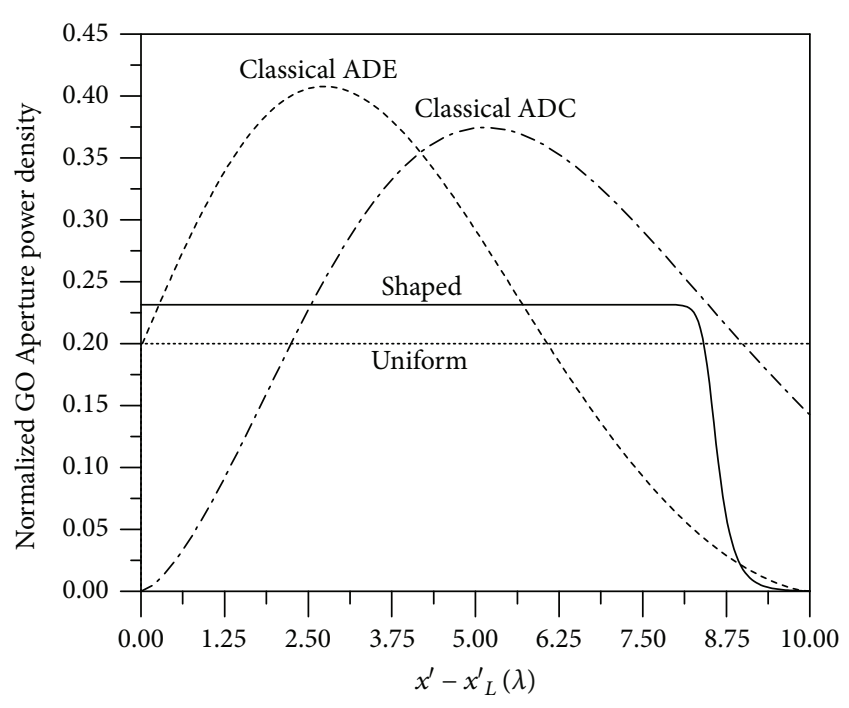

FIgURE 13: GO aperture power density distributions $G\left(x^{\prime}\right)$.

Figures 7 and 11 for $140^{\circ}<\theta<180^{\circ}$, while maintaining a desirably large main-beam directivity. As mentioned before, such high side lobe levels are mainly due to the spillover from the main reflector. So, instead of shaping the reflectors with uniform amplitude at the conical aperture, an alternative amplitude distribution was tailored to reduce the illumination toward the main reflector edge:

$$
G\left(x^{\prime}\right)= \begin{cases}G_{0}, & \text { for } x_{L}^{\prime} \leq x^{\prime} \leq x_{o}^{\prime}, \\ G_{0}\left\{\left[\frac{2\left(x^{\prime}-x_{o}^{\prime}\right)}{\sigma_{1} W_{A}}\right]^{\sigma_{2}}+1\right\}^{-2 \sigma_{3}}, & \text { for } x_{o}^{\prime} \leq x^{\prime} \leq x_{U}^{\prime},\end{cases}
$$

where $x_{0}^{\prime}=\left(x_{L}^{\prime}+x_{U}^{\prime}\right) / 2$ is at the aperture center, $\sigma_{1}, \sigma_{2}$, and $\sigma_{3}$ are the constants that control the aperture power density, and $G_{0}$ is a normalization factor. Both configurations, ADE and $\mathrm{ADC}$, were resynthesized with $\sigma_{1}=0.7, \sigma_{2}=40$, and $\sigma_{3}=$ 0.242088 to provide an edge attenuation of $-30 \mathrm{~dB}$ at $x^{\prime}=$ $x_{U}^{\prime}$. Figure 13 illustrates the power distribution at the aperture for the several cases investigated in this work. Besides the change in the aperture amplitude $G\left(x^{\prime}\right)$, the input parameters used in the previous (uniform amplitude) ADE and ADC shaping cases were kept the same.

Figures 14 and 15 show the reflectors' generatrices of the shaped $\mathrm{ADE}$ and $\mathrm{ADC}$ configurations, respectively, with $\mathrm{G}\left(x^{\prime}\right)$ given by (15), together with ray tracings. Comparing these results with those from Figures 5 and 9, one readily observes the significant reduction in the density of rays toward the main reflector edge, as desired. Figures 16 and 17 show MoM/MMT radiation patterns for all the $\mathrm{ADE}$ (Figure 16) and ADC (Figure 17) antennas investigated in this work. From these figures, one observes that the synthesis yielded designs with high gains, close to those obtained by the uniform distribution (12.68 $\mathrm{dBi}$ for the ADE and $12.59 \mathrm{dBi}$ for the ADC). Besides, as desired,

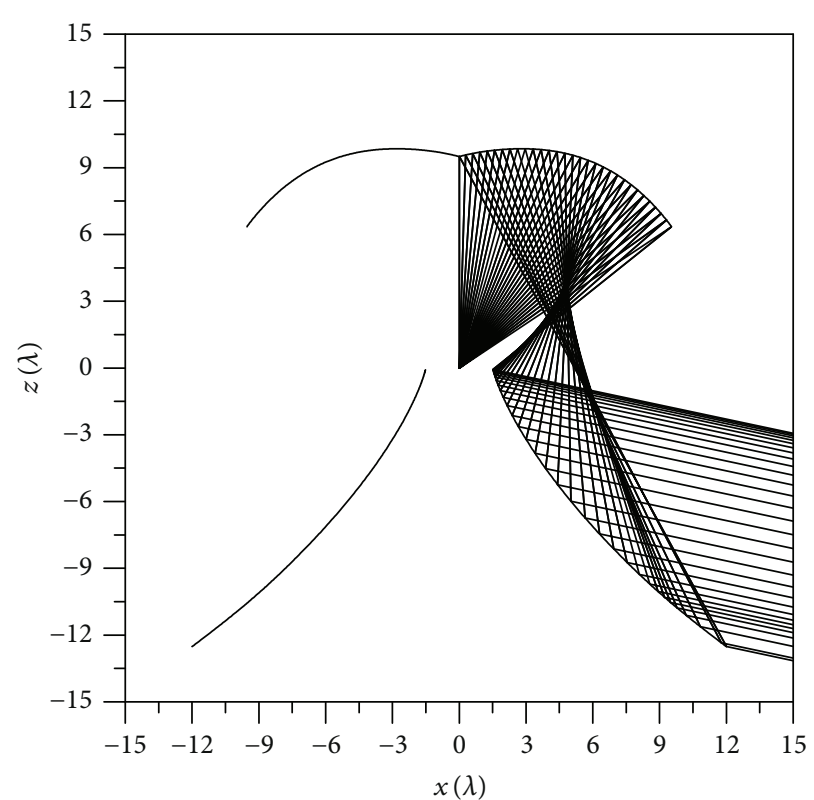

FIgURE 14: Generatrices and ray tracing of the shaped ADE antenna $\left(-30 \mathrm{~dB}\right.$ attenuation at $\left.x_{U}^{\prime}\right)$.

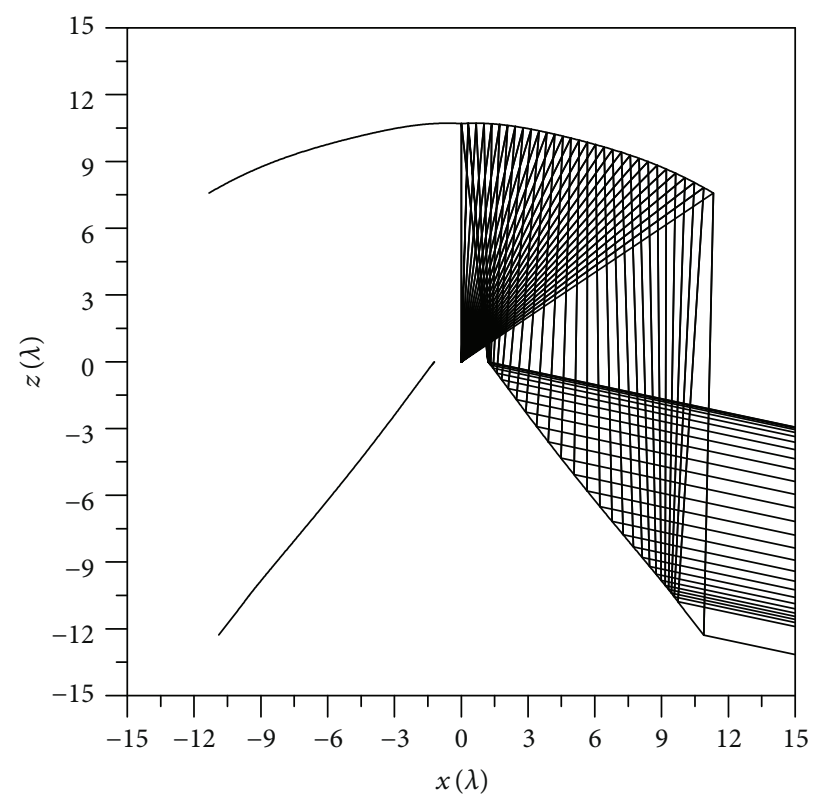

FIgURE 15: Generatrices and ray tracing of the shaped ADC antenna $\left(-30 \mathrm{~dB}\right.$ attenuation at $\left.x_{U}^{\prime}\right)$.

a reduction in the side lobe levels in the region below the main reflector $\left(140^{\circ}<\theta<180^{\circ}\right)$ was attained, especially for the ADC case.

Figure 18 shows the maximum errors for the shaped sub- and main reflectors of Figures 14 (ADE) and 15 (ADC) as a function of $N$. Again, due to the cumulative effect, the maximum errors occur at reflector points associated to $\theta=\theta_{E}$. Designs with $N=20$ show maximum error of $0.004 \lambda$ and RMS error of $0.003 \lambda$ for the ADE-like configuration and maximum error of $0.018 \lambda$ and RMS error of $0.005 \lambda$ for the ADC-like configuration. 


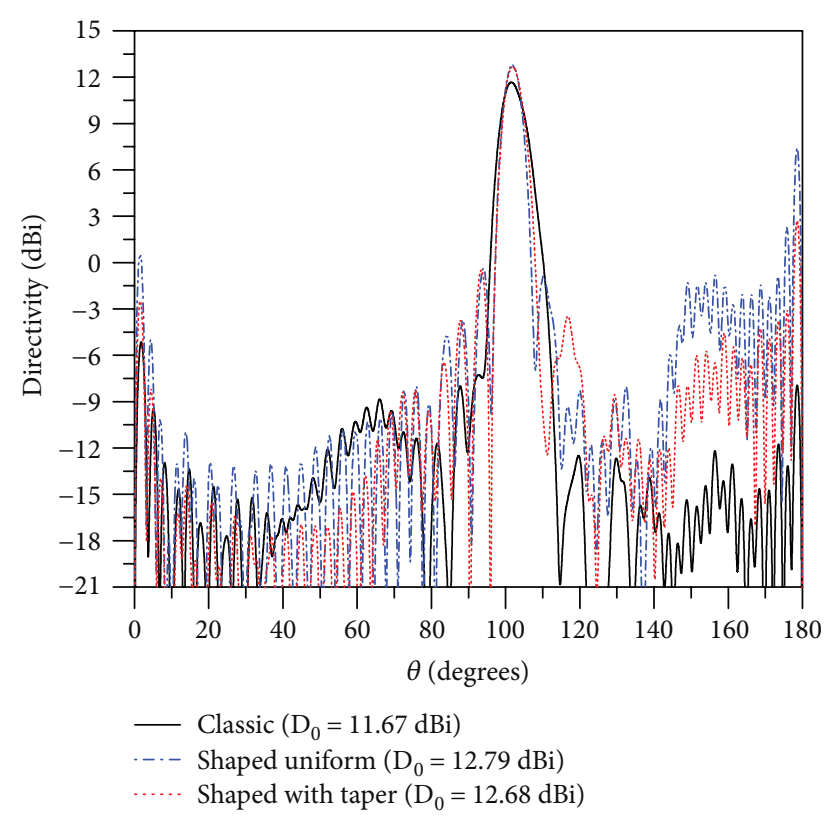

FIgUre 16: MoM/MMT radiation patterns of the classical and shaped ADE antennas.

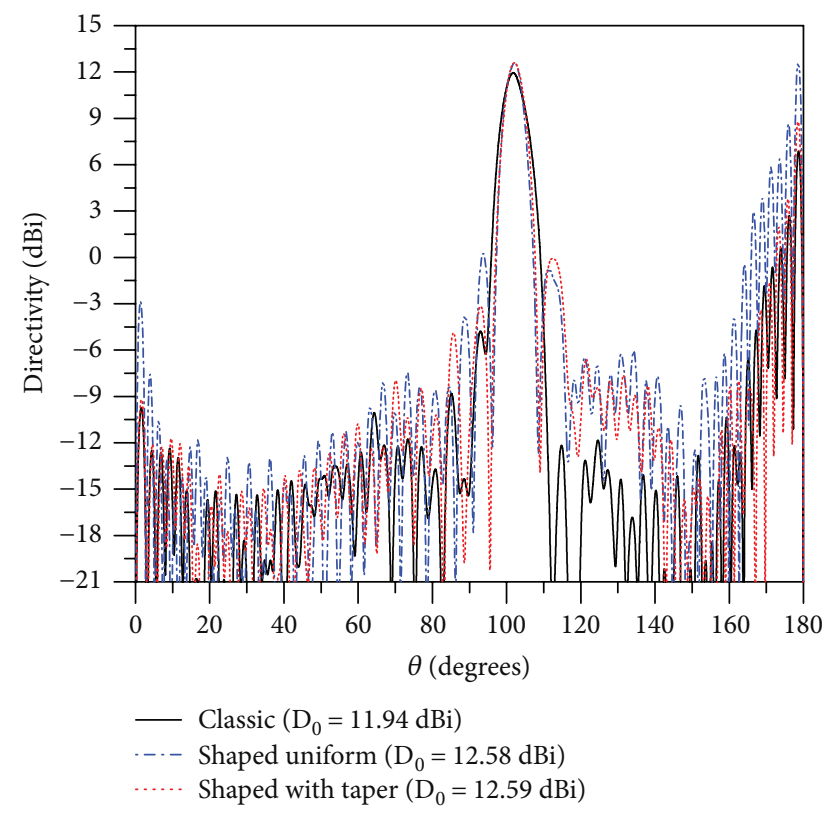

Figure 17: MoM/MMT radiation patterns of the classical and shaped ADC antennas.

\section{Conclusions}

A method for the GO synthesis of omnidirectional dualreflector antennas with equiphase field in a conical aperture has been presented. The sub- and main reflector generatrices were represented by the consecutive concatenation of local conic sections, and, by imposing geometrical optics principles, their shapes were obtained by solving liner equations. Omnidirectional ADE- and ADC-like configurations were successfully designed for uniform and tapered power

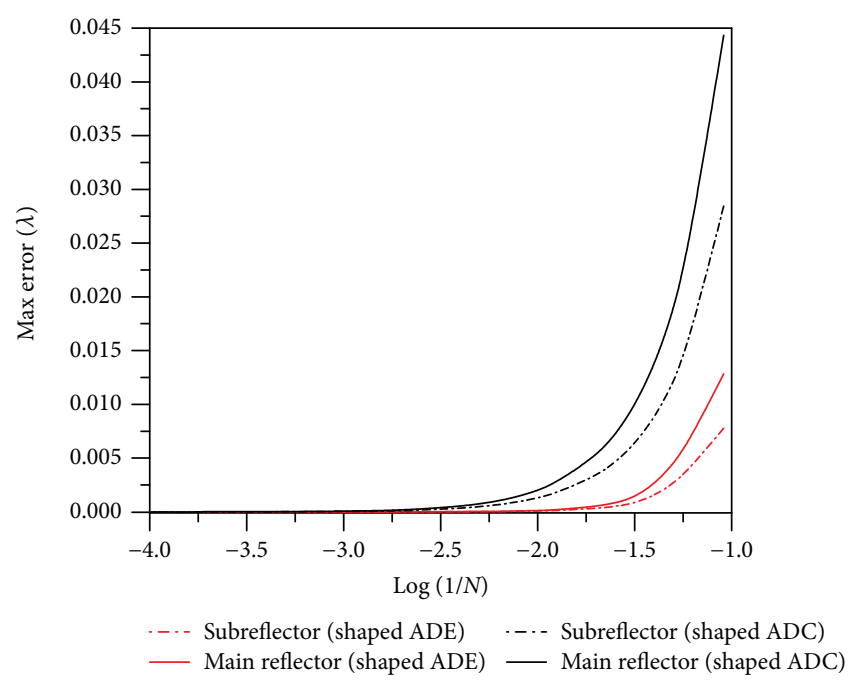

Figure 18: Maximum error of shaped ADE and ADC antennas $\left(-30 \mathrm{~dB}\right.$ attenuation at $\left.x_{U}^{\prime}\right)$ as a function of $N$.

distributions at the equiphase conical aperture. The concatenation of conic sections allowed an efficient representation of the shaped reflectors, with numerical convergence similar to that observed in [7]. The antenna design examples were fed by a coaxial TEM horn, and their performance were validated by employing a rigorous analysis technique that combines the mode matching technique (MMT) and the method of moments (MoM), which account for all electromagnetic effects present on the reflector and horn structure.

\section{Conflicts of Interest}

The authors declare that they have no conflicts of interest.

\section{Acknowledgments}

This work was partially supported by FINEP/FUNTTEL Grant no. 01.14.0231.00, under the Radio Communications Reference Center (CRR), CAPES-PROCAD Grant 068419/ 2014-01, CNPq, and FAPEMIG.

\section{References}

[1] T. Rappaport, S. Sun, R. Mayzus et al., "Millimeter wave mobile communications for 5G cellular: it will work!," IEEE Access, vol. 1, pp. 335-349, 2013.

[2] V. Galindo, "Design of dual-reflector antennas with arbitrary phase and amplitude distributions," IEEE Transactions on Antennas and Propagation, vol. 12, no. 4, pp. 403-408, 1964.

[3] A. G. Pino, A. M. A. Acuña, and J. O. R. Lopez, "An omnidirectional dual-shaped reflector antenna," Microwave and Optical Technology Letters, vol. 27, no. 5, pp. 371-374, 2000.

[4] F. Moreira, A. Prata Jr., and J. R. Bergmann, "GO shaping of omnidirectional dual-reflector antennas for a prescribed equi-phase aperture field distribution," IEEE Transactions on Antennas and Propagation, vol. 55, no. 1, pp. 99-106, 2007.

[5] Y. Kim and T.-H. Lee, "Shaped circularly symmetric dual reflector antennas by combining local conventional dual 
reflector systems," IEEE Transactions on Antennas and Propagation, vol. 57, no. 1, pp. 47-56, 2009.

[6] P.-S. Kildal, "Synthesis of multireflector antennas by kinematic and dynamic ray tracing," IEEE Transactions on Antennas and Propagation, vol. 38, no. 10, pp. 1587-1599, 1990.

[7] F. Moreira and J. Bergmann, "Shaping axis-symmetric dual-reflector antennas by combining conic sections," IEEE Transactions on Antennas and Propagation, vol. 59, no. 3, pp. 1042-1046, 2011.

[8] R. Penchel, J. Bergmann, and F. Moreira, "Main-reflector shaping of omnidirectional dual reflectors using local conic sections," IEEE Transactions on Antennas and Propagation, vol. 61, no. 8, pp. 4379-4383, 2013.

[9] F. Moreira and J. Bergmann, "Omnidirectional dual-reflector shaping by concatenating conic sections," in Proceedings of the Fourth European Conference on Antennas and Propagation, Barcelona, Spain, April 2010.

[10] S. Zang and J. Bergmann, "Analysis of omnidirectional dualreflector antenna and feeding horn using moment methods," IEEE Transactions on Antennas and Propagation, vol. 62, no. 3, pp. 1534-1538, 2014.

[11] F. Moreira and J. Bergmann, "Axis-displaced dual-reflector antennas for omnidirectional coverage with arbitrary mainbeam direction in the elevation plane," IEEE Transactions on Antennas and Propagation, vol. 54, no. 10, pp. 2854-2861, 2006. 


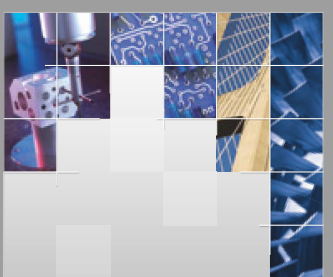

\section{Enfincering}
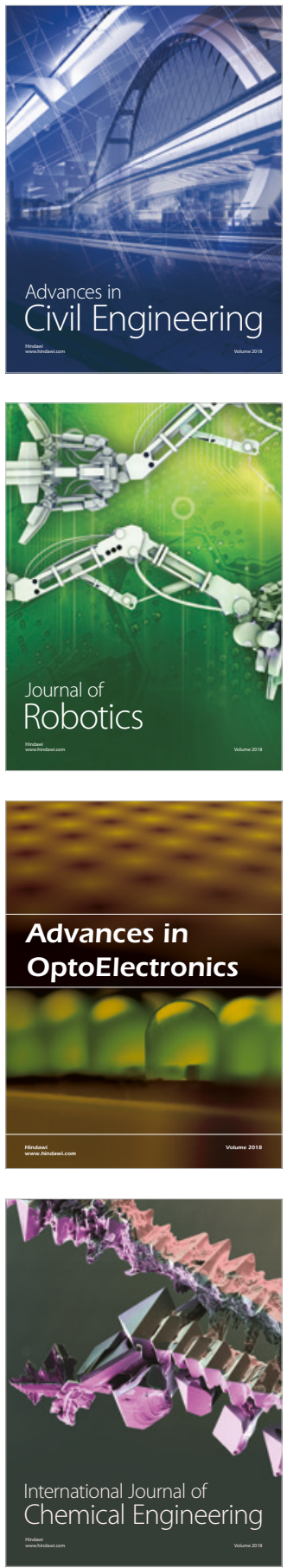

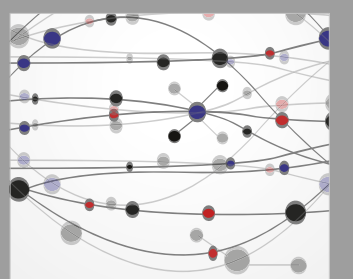

\section{Rotating \\ Machinery}

The Scientific World Journal

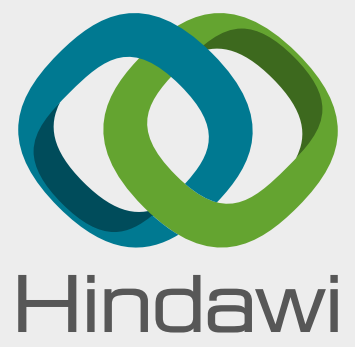

Submit your manuscripts at

www.hindawi.com
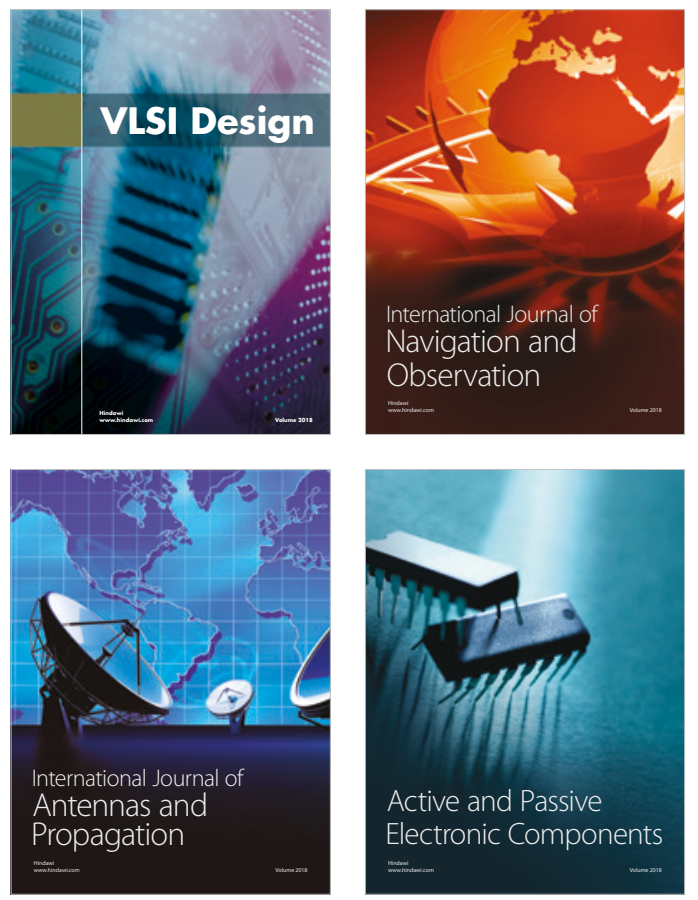
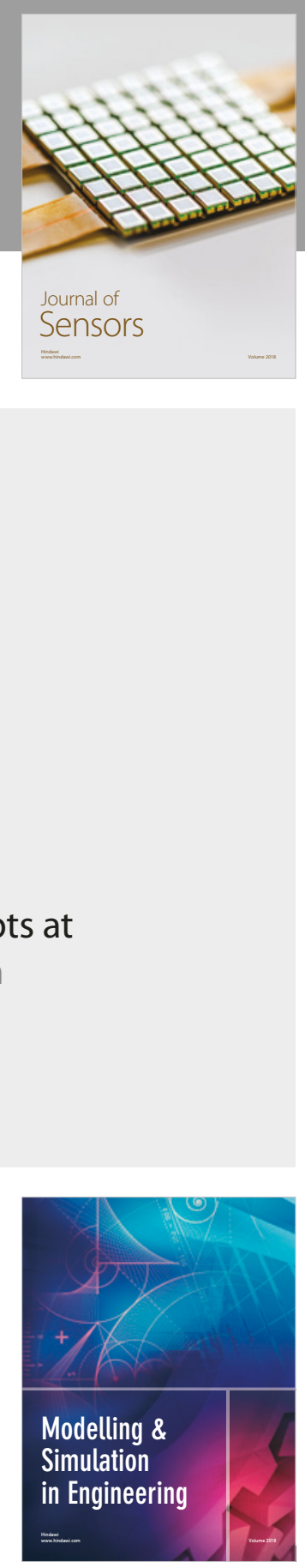

\section{Advances \\ Multimedia}
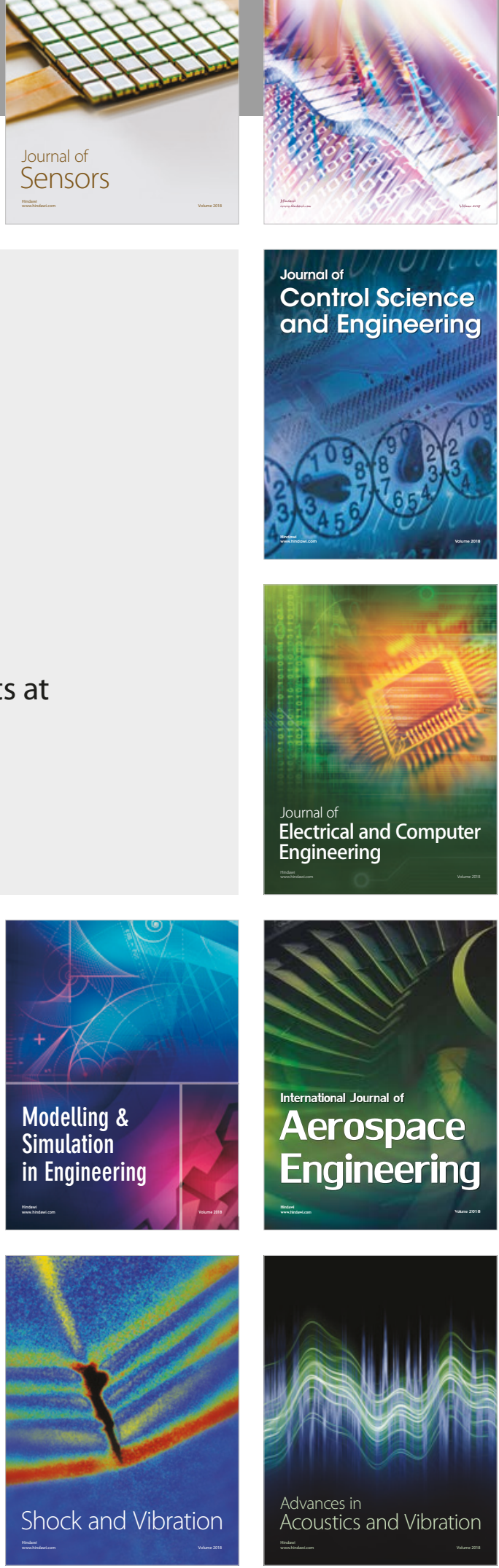